\title{
Waste LED lamp characterization: A call for more consistent and well-documented study
}

\author{
SM Mizanur Rahman ${ }^{\mathrm{a}}$, Bertrand Laratte ${ }^{\mathrm{b}, \mathrm{c}, \mathrm{d}, \mathrm{e}}$, Stéphane Pompidou ${ }^{\mathrm{a}}$, Thècle Alix ${ }^{\mathrm{b}}$
}

\author{
${ }^{\text {a }}$ University of Bordeaux, CNRS, Arts et Metiers Institute of Technology, Bordeaux INP, INRAE, I2M \\ Bordeaux, F-33400 Talence, France \\ ${ }^{b}$ Arts et Metiers Institute of Technology, University of Bordeaux, CNRS, Bordeaux INP, INRAE, I2M \\ Bordeaux, F-33400 Talence, France \\ ${ }^{c}$ Department of Industrial Engineering, Ondokuz Mayıs University, 55139 Samsun, Turkey \\ ${ }^{d}$ APESA-Innovation, F-40220 Tarnos, France \\ ${ }^{\mathrm{e}}$ French Institute of Anatolian Studies, CNRS USR 3131, Palais de France Nur-i Ziya, sk. 10 PK 54 \\ 34433 Beyoğlu, Istanbul, Turkey
}

\section{Abstract}

Solid-state lighting has been replacing conventional lighting in the market, raising concerns for implementing an efficient end-of-life management system. Since LED waste streams have not been quite dominant, characterization studies (product-level characteristics, componentwise information, bill of materials, diversity, and differences) are inconsistent and having substantial gaps. This study investigates the end-of-life LED lamp literature, focusing on the bill-of-materials, and reveals that the characterization procedure suffers from a severe lack of sample representativeness. Consequently, characterization results are widely varied and not generalizable. Moreover, most of the studies did not inform and document the detailed sample characteristics, precluding the possibility of identifying reasons for variation. Combining available information from the recent studies, we present a mass distribution at the component level, exemplifying the prospect for meeting EU regulatory limit and the need for more insight at the lamp and component level. To fill the disconnection between lamp level characteristics and component and material content, we propose a characterization protocol that carefully documents lamp, component, and material level information by establishing a lamp-component-material composition nexus. The protocol may help researchers, policymakers, and industrial stakeholders conduct a systematic characterization, analyze complexities and prepare for a sustainable solution.

Keywords

Solid waste management, LED lamp, characterization protocol, recycling, LED waste management

\subsection{Introduction}

The LED lamp recycling is undergoing a critical juncture when recyclers, decision-makers, and researchers desperately look for a sustainable recycling line. At the same time, an understanding of the waste stream is still limited. The characterization approach undertaken by the industrial and non-industrial practitioners suggests a dilemma as to whether early segregation or a direct crushing approach would be more suitable for treating LED lamps. The early segregation at the component level tends to avoid mixing of the precious and Rare Earth Elements (REEs), which, in turn, enhances the possibility of the purer bulk material recovery 
as well as the prospect for precious and REEs recovery (LED professional 2016, Cenci et al. 2020, Martins et al.2020). However, technology for early segregation is not quite optimal at this moment. Alternatively, direct crushing tends to employ the traditional shredding and sorting-based approach and optimize parameters to increase yield. This approach can not meet the regulatory limit by a margin of about $20 \%$, including precious metals and REEs, destined to landfill. In addition, a fraction of the precious metals and REEs hide in the recoverable fractions as impurities that the available sorting techniques can seldom eliminate, resulting in less pure bulk fractions (LED professional 2016).

This debate is going on in the context of less-than-enough information regarding the waste stream at the lamp, component, and material level. We present two findings: 1) Kumar et al. (2018) conducted high-voltage-pulse-based comminution on the eight lamps, classifying lamps into three categories based on body types. They found that the comminution process successfully fragments the plastic casing lamps but could not disintegrate metal casing lamps (three out of eight). The other lamp groups - glass casing lamps - yielded too many fines. They concluded that metal and glass casing lamps are not worthy for a coarse fragmentation process. 2) Lim et al. (2011) performed Total Threshold Limit Concentration (TTLC) tests on nine pin-type LED chips, different by color and intensity. After investigating toxicity levels, they have found that white LED chips are safer while others pose various adverse health and environmental impacts.

These two findings stress that lamp and component level features can potentially be essential for any subsequent decision-making related to end-of-life treatment. This paper aims to initiate a discussion if the information at the lamp, component and material level can potentially help select a resource-efficient recycling line. The assumption is that the lampcomponent-material composition nexus may provide important insight by exploiting the various lamp feature, which critically determines which materials to be recovered, at what level, and how. To further investigate the topic, we explore LED lamp literature to collect available information on lamp, component, and material level and discuss how a more systematic procedure should complement the current approach.

\subsection{Lamp feature and bill of materials:}

An LED Lamp can be classified based on its external features. This study has considered four external features of LED lamps: body type, shape type, base type, and applications. An LED lamp can be in three categories based on the body type: metal casing, plastic casing, and glass casing. Categories based on shape type can be many, but most lamps fall into six categories: A series, G series, PAR series MR series, R series, and T series. Base type category can also be many, but E27, E14, GU10, Bi-pins, and B22 cover most waste lamps. Applications-based categories can be industrial, commercial, residential, and architectural. Overall, these categories are showing the diversity in lamp level. Many other lighting categories are entering every day, indicating the dynamic nature of the lamp level categories.

However, the current studies did not capture the dynamics. Rahman et al.(2019) performed a lamp-level characterization, for which a sample size of $414 \mathrm{~kg}$ (3029 units) is used (Rahman et al., 2019). The results showed that about $90 \%$ of the total sample were one of the three base categories E27 (30\%), E14 (32\%), and GU10 (30\%). Similarly, among the six shape categories, the A-series (30\%), G series (30\%), and PAR series $(20 \%)$ accounted for more than $80 \%$ of the lamp types (Rahman et al., 2019). Table (1) highlights gaps in studies related to the product level feature. For example, PCBs and LED chips are glued in some lamps, affecting the liberation (Dzombak et al., 2017). However, their proportion in the LED lamps 
is not quantified, nor are they discussed how they could be identified and processed. Similarly, body type is an essential variable that, as pointed out above, can potentially be crucial for selecting the recycling line but is not studied closely (Kumar et al., 2018). A study quantifies the distribution by base type and shape type, but future studies should repeat for different times, countries, and samples. Moreover, the study did not discuss if the difference of lamp categories impacts the component and material recovery.

Table 1. Product characteristics analysis in studies

\begin{tabular}{ll}
\hline Product characteristics & References \\
\hline Base type & (Rahman et al., 2019) \\
Shape type & (Rahman et al., 2019) \\
Body type (plastic or metal, or glass) & No study \\
PCB and LED attached or separated & No study \\
Application (industrial, commercial or residential) & No study \\
Brand type & (Dzombak, 2017) \\
Year of sold & (Dzombak, 2017) \\
\hline
\end{tabular}

\subsection{Component- bill of material connection}

Component-level categories are also quite diverse. Hendrickson et al. (2020) propose seven categories: Edison screw, driver, base assembly, heat sink, LED module, housing, and optics. Dzombak et al. (2017) aim to align with Hendrickson et al. (2020), dividing the components into seven categories with some modifications (diffuser $=$ optics, power supply $=$ driver, base assembly $=$ potting material) (Dzombak et al., 2017). Martins et al.(2020) classified the components into nine categories (Edison screw, polymeric cover, polymeric housing, metallic housing, PCB, heat sink with LEDs, capacitors, coils, and wires), which is similar to the four categories in Cenci et al. (2020): the carcass, PCBs, LED module and LEDs (Cenci et al., 2020; Martins et al., 2020).

The combination of the composition information gives greater detail of the types of components and their mass distribution. For comparison, polymeric cover, polymeric housing, metallic housing, and base constitute the carcass in Cenci et al. (2020), which share $70 \%$ of the total weight of a LED lamp. The rest includes PCBs (» 15\%) and the LED module with the heat sink (» 9\%) and the base (»7\%). Among the 70\%, polymeric cover/optics-plastic/diffuser is the top part of a LED lamp, which weighs about $20 \%$ of the overall mass, and polycarbonate is the only plastic material used as an optical diffuser (Martins et al., 2020). Other studies do not specifically mention the type of plastic material used as a diffuser. In general, the industries use polycarbonate as an excellent optical diffuser. Polymeric housing constitutes about $28 \%$ of the mass, which contains either polyethylene terephthalate (PET) and polybutylene terephthalate (PBT) (Martins et al., 2020) or polyamide and polyester (Cenci et al., 2020). The metallic housing shares about $14 \%$ of the mass, and the Edison screw represents about $7 \%$. However, it is unclear whether some lamps utilize both metal and plastic housing for thermal management. It is also essential to know if all lamps use the same polymer consistently. The type of information currently lacks in the studies covered. The weight of PCB and electronic parts have also been estimated by Martins et al.: printed circuit boards (PCBs) $(5 \%)$ and capacitors $(7 \%)$, coil (6\%), and wires (1.4\%) (Martins et al., 2020). LEDs with heat sink varies in the range of 3.2 to $12 \%$, with the LED chip fraction ranging from 0.32 to $0.69 \%$ (figure 1 ) 
The composition of dominant material in some components indicates the possibility of recovering fractions with high purity. The Edison screw consists of $82 \%$ iron and $12 \%$ nickel, the polymeric cover about $100 \%$ polycarbonate, the metallic housing about $95 \%$ aluminum, the capacitor is $84 \%$ aluminum, and the coil is $92 \%$ copper (Martins et al., 2020). These metals make up $78 \%$ of the total mass, with average purity not below $80 \%$ (Table S2). The componentbased recovery can, therefore, (or be closed to) meet the EU regulation. Material-wise, about $27 \%$ of the metals are copper, aluminum, and iron; $20 \%$ are polycarbonate, and about $28 \%$ are one or all of the four types of plastics. Early segregation based on the component can facilitate the recovery of high-quality recyclate fractions (Figure 1). The analysis is based on a minimum sample size, requiring more samples to validate.

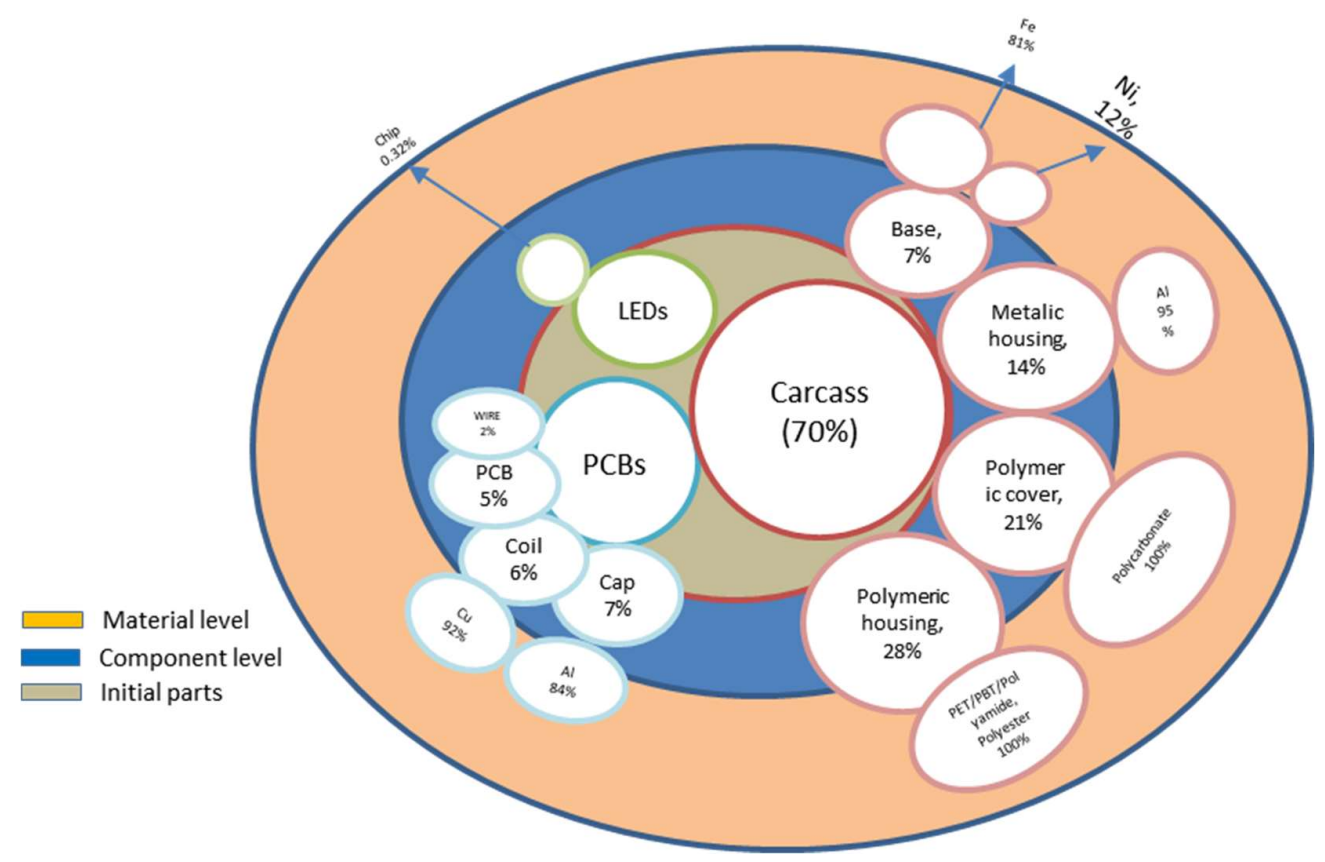

Figure 1. The component-material composition connection

\subsection{PCB and LED module-bill of material connection:}

LED modules and PCBs (along with electronic parts) constitute no more than $25 \%$ of the total mass of LED lamp weight but comprise about 20 different materials. They are specifically crucial for their precious and strategic material content. As mentioned before, the minor metals in these components, unless separated, get mixed (and lost) with the recoverable fractions, which reduces the purity of the recoverable fractions.

Only a few studies have characterized PCB and electronic parts of LED lamps. As mentioned before, the share of PCBs in the LED lamp varies from 15 to 20\%. The metal portion (58\%) comprises about 17 materials (the rest are polymer 30\% and ceramics 12\%) (Zamprogno Rebello et al., 2020). Aluminum, antimony, arsenic, cerium, copper, gallium, gold, iron, lead, nickel, silver, tin, titanium, and yttrium are the metals reported in the metal portion. However, Cenci et al. (2020) did not report antimony, arsenic, cerium, lead, and yttrium. Rebello et al. (2020) use a larger sample with 16 different models than three lamps in Cenci et al. (2020). The concentration of the materials reported also varies. Aluminum is the leading metal occupying $110 \mathrm{~g} \cdot \mathrm{kg}-1$, followed by copper with $94 \mathrm{~g} \cdot \mathrm{kg}-1$. In Cenci et al. (2020), copper is 
the central metal with $26.1 \mathrm{~g} \cdot \mathrm{kg}-1$, followed by aluminum with $9.1 \mathrm{~g} \cdot \mathrm{kg}-1$ (Cenci et al., 2020). Similar differences are also observed in gold and silver content, which are about ten and one hundred times higher in Zamprogno Rebello et al. (2020) than in Cenci et al. (2020), respectively. Gallium is identified in Cenci et al. (2020) but not in Zamprogno Rebello et al (2020). In Cenci et al. (2020), 79\% gallium is found in PCBs, contrary to the general belief that gallium is mainly used in LED chips (Table S3).

The characterization of LED modules is performed in few studies. Different components of the LED module include the LED die, the package, and the module. The LED die is composed of arsenic, gallium, and indium. The interconnection technologies in the LED package contain gold and silver, and phosphor contains rare earth metals. LED module contains copper, while solder contains gold and silver. Other compounds contain aluminum, manganese, zinc, and palladium (Table S4).

Lim et al. (2011) performed TTLC and toxicity characteristics leaching procedure (TCLP) tests to examine the metallic content of 9-pin type LED chips, which differ by color and intensity: red/low-intensity, red/high, yellow/low, yellow/high, green/low, green /high, blue/low, blue/high and white (Lim et al., 2011). Aluminum, antimony, arsenic, chromium, copper, gallium, gold, iron, nickel, silver, and zinc were found in each sample, whereas barium, cerium, gadolinium, indium, lead, mercury, phosphorus, tungsten, and yttrium were inconsistently identified. According to this study, copper, lead, nickel, iron, and silver exceed California state regulations, while only lead exceeds the federal regulations. High toxicity potentials are reported for arsenic, copper, nickel, lead, iron, and silver. High resource depletion potentials are reported for gold and silver. Overall, white LEDs are considered the safest due to their low copper content and lack of lead and arsenic. Red low-intensity LEDs have shown high cancer and non-cancer potential. High-intensity LEDs show high toxic potential due to the presence of copper and arsenic. High variation is observed for copper in the range of 0.032 to $3.892 \mathrm{~g} \cdot \mathrm{kg}-1$. The lowest is found in white LEDs, while the highest is found in low-intensity blue LEDs. A similar difference is also observed in two studies of the same authors with 25.35 and $928.93 \mathrm{mg} \cdot \mathrm{kg}$-1, respectively (Cenci et al., 2021, 2020). For iron, the concentration varies from 256 to $358 \mathrm{~g} \cdot \mathrm{kg}$-1, but in Cenci et al. (2020), the value is only $0.05 \mathrm{mg} \cdot \mathrm{kg}-1$ or less (Cenci et al., 2021, 2020). The gold concentration varies from $0.003 \mathrm{~g} \cdot \mathrm{kg}-1$ to $0.176 \mathrm{~g} \cdot \mathrm{kg}-1$ in high-intensity green LEDs. The gold concentration in white LEDs is also recorded at a moderately high level $(0.115 \mathrm{~g} \cdot \mathrm{kg}-1)$ (Cenci et al., 2020). In Cenci et al. (2021), it is $0.82 \mathrm{mg} \cdot \mathrm{kg}-1$ (Table S5).

Although not identified in all of the different LEDs, the content of toxic elements must be confirmed by the larger sample. Arsenic is found in all samples except the white LEDs. Lead is found in four of the nine LEDs and poses a risk to human and environmental wellbeing. Unsurprisingly, mercury is not detected in any of the LEDs. Cenci et al.(2021) detect no lead, and arsenic or Cenci et al. (2020) detect low value.

\subsection{Discussion:}

Most studies, so far, have been based on either a limited number of LED lamps (less than five) or the sample size unmentioned (Zhan et al., 2015) ( see detail in table S1). The larger sample, $600 \mathrm{~kg}$, and $11.4 \mathrm{~kg}$ is only a recent trend (Kumar et al., 2019; Zamprogno Rebello et al., 2020). A couple of studies have performed TCLP tests, with a sample of $600 \mathrm{~kg}$ and only one LED lamp, respectively. Zamprogno Rebello et al. (2020) have analyzed $1.4 \mathrm{~kg}$ of PCB samples, while Cenci et al.(2020) and Martins et al. (2020) have analyzed only three and five LED lamps, respectively. Despite the smaller sample size, Dzombak et al. (2017) detailed the 
product sample extensively enough to be reproducible for other studies. The product name, brand, model, year of marketing, luminous flux (in lumen), lifespan, and price of the lamps were recorded and correlated with their component and material content. The sample is then manually disassembled to identify the disassembly challenges. The detailed characterization (mentioning the year of marketing) permits, for example, temporal analysis of whether the design suggestion has been integrated into the subsequent year's product.

Although a generalization based on the studies provides a relatively better understanding of the component-material connections, the studies often report a significant variation in the components' weight percentage. We have analyzed 17 A19 lamps (Dzombak et al., 2017) and three A19 lamps (Hendrickson et al., 2010) with five lamps from Martins et al.(2020) and three lamps from Cenci et al.(2020). The polymeric cover shows a variation from 0 to $40 \%$. Some studies did not report any polycarbonate, while some report weight percentage of polycarbonate up to $40 \%$. Similarly, the polymeric housing varies from 0 to $65 \%$, reflecting PET/PBT vastly varied. Similar variations are also observed from LED modules, PCBs, Edison screw base, and others (Table 2). The variation points to the differing types of LED lamps (e.g., plastic casing or metal casing) and a different set of components in each of the LED lamps.

Table 2. LED lamp composition variation among different studies

\begin{tabular}{|c|c|c|}
\hline & Variation (\%) & Dominant material \\
\hline Polymeric cover & $0-40$ & Polycarbonate \\
\hline Polymeric housing & $0-65$ & $\begin{array}{l}\text { PET / PBT / Polyamide } \\
\text { / polystyrene }\end{array}$ \\
\hline Metallic housing & $0-25$ & $95 \%$ aluminum \\
\hline LED module & $2.6-12$ & - \\
\hline LEDs & $0.32-0.69$ & - \\
\hline PCBs & $5-15$ & - \\
\hline Driver & - & - \\
\hline Base & $2-10$ & $82 \%$ iron \\
\hline
\end{tabular}

In addition to the problem of the limited sample size, inconsistent reporting is also observed. In Lim et al.(2011,) no plastic is reported, while approximately $95 \%$ aluminum is reported. Kumar et al. (2019) report a total of $31 \%$ of the mass, which excluded plastic. The aluminum content is about $22 \%$. Five common metals ( $\mathrm{Al}, \mathrm{Fe}, \mathrm{Ni}, \mathrm{Cu}$, and $\mathrm{Ag}$ ) are identified in all four studies, while the remaining 14 elements were inconsistently recorded (Table 3 ). Gold is present in all studies except Kumar et al.(2019). Mercury is detected in Lim et al. (2012), while lead is detected in both Kumar et al. and Lim et al. (Kumar et al., 2019; Lim et al., 2012). Gallium and indium are also not consistently reported. Among REEs, cerium and yttrium are mentioned but not identified in all studies. Thus, it is not possible to conclude from the studies whether or not certain REEs or hazardous metals are specific to a particular type of LED lamp. 
Table 3. Comparison of the different studies (Cenci et al., 2020; Kumar et al., 2019; Lim et al., 2012; Zamprogno Rebello et al., 2020)

\begin{tabular}{|c|c|c|c|c|}
\hline & $\begin{array}{l}\text { Kumar et al. } \\
(2019)\end{array}$ & $\begin{array}{l}\text { Lim et al. } \\
\text { (2013) }\end{array}$ & $\begin{array}{l}\text { Cenci et al. } \\
(2020)\end{array}$ & $\begin{array}{l}\text { Rebello et al. } \\
(2020)\end{array}$ \\
\hline Plastic & Not reported & $\begin{array}{l}\text { Not } \\
\text { reported }\end{array}$ & $70 \%$ & n.a. \\
\hline Scope & Metal -31\% & $\begin{array}{l}\text { LED chip- } \\
100 \%\end{array}$ & $30 \%$ & $\begin{array}{l}\text { PCB only- } \\
13 \%\end{array}$ \\
\hline Arsenic & Reported & $\begin{array}{l}\text { Not } \\
\text { detected }\end{array}$ & $\begin{array}{l}\text { Not } \\
\text { detected }\end{array}$ & Reported \\
\hline $\begin{array}{l}\text { Common metals } \\
\text { detected } \\
\text { (Al, } \mathrm{Fe}, \mathrm{Ni}, \mathrm{Cu} \text {, and } \\
\text { Silver) }\end{array}$ & Reported & Reported & Reported & Reported \\
\hline Mercury & Not detected & Reported & $\begin{array}{l}\text { Not } \\
\text { detected }\end{array}$ & $\begin{array}{l}\text { Not } \\
\text { detected }\end{array}$ \\
\hline Gallium & Not detected & Reported & Reported & $\begin{array}{l}\text { Not } \\
\text { detected }\end{array}$ \\
\hline Indium & Reported & $\begin{array}{l}\text { Not } \\
\text { detected }\end{array}$ & Reported & $\begin{array}{l}\text { Not } \\
\text { detected }\end{array}$ \\
\hline Gold & Not detected & Reported & Reported & Reported \\
\hline Cerium & Not detected & Reported & Reported & Reported \\
\hline Yttrium & Not detected & Reported & Reported & Reported \\
\hline Lead & Reported & Reported & $\begin{array}{l}\text { Not } \\
\text { detected }\end{array}$ & $\begin{array}{l}\text { Not } \\
\text { detected }\end{array}$ \\
\hline Antimony & Reported & Reported & $\begin{array}{l}\text { Not } \\
\text { detected }\end{array}$ & Reported \\
\hline Chromium & Reported & Reported & $\begin{array}{l}\text { Not } \\
\text { detected }\end{array}$ & Reported \\
\hline $\begin{array}{l}\text { Calcium and } \\
\text { Manganese }\end{array}$ & Reported & $\begin{array}{l}\text { Not } \\
\text { detected }\end{array}$ & $\begin{array}{l}\text { Not } \\
\text { detected }\end{array}$ & $\begin{array}{l}\text { Not } \\
\text { detected }\end{array}$ \\
\hline
\end{tabular}

We have found that few attempts investigated the lamp level feature. The majority of the studies randomly selected a specific type of lamp/s without considering representativeness, which reduces the validity and reliability of the result. In addition, the result loses essential insight. For example, we can not analyze if any difference in the distribution of lamp categories would influence the rate and types of material recovery. There is no systematic information as to how the lamp distribution by categories varies over time. To improve and document the characterization results, the following figure 2 can be a good example. In the left column, we can include lamp categories, mentioning their relative contribution. Similarly, the other columns, component, fraction, and material level information can be added. The presentation of this type can help understand the lamp-component-material composition nexus. 


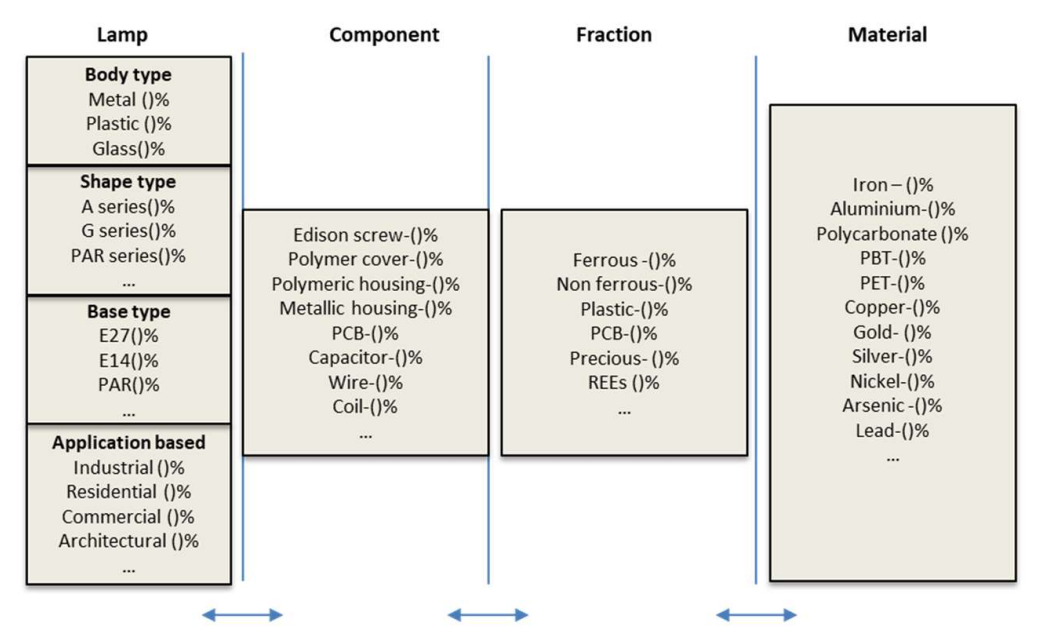

Figure 2: A possible documentation framework of the characterization studies

\subsection{Characterization protocol}

To ensure the sample selection and documentation consistency, we propose a characterization protocol (Figure 3). At lamp level, a researcher first determines if the sample size is large enough, considering a ratio of the sample relative to the total waste streams of the facility in the year and incorporating all of the dominant types of lamps in the corresponding proportion (Scdhec 2021). A researcher provides a detailed explanation of how the sample corresponds to the current waste stream of the region. If the initial sampling is justified, a lamp-level characterization can be performed by categories more closely. The characterization features may include - but are not limited to - body type, base, shape, year of manufacturing, brand name, and application. The product characterization step will provide further information to a researcher if the sample represents each category proportionately, without much bias to one or few features. If so, more lamps of features missing in the sample can be added to be better representative, or else, a researcher can justify the under-representation.

Once the product characterization is complete, a preliminary observation can be performed at the component level (e.g., any new devices are noticed). If any changes are observed, the sample should be re-analyzed by asking questions if such changes are significant or an exception. If it deems that the change reflects a new trend in the waste streams, then the sample should be revised, adding the necessary categories, and the subsequent steps should be followed. Then a manual or mechanical disassembly can be conducted for a component-level characterization. The challenge, in reality, is that the large sample may not allow manual sorting of the components due to time and resources. However, the distribution of the components should be documented to the extent possible. Next, a shredding and sorting operation can be performed on each component, and then a fraction level analysis can be performed.

Alternatively, a component-level operation can be skipped for a direct shredding and sorting process, which yields different fractions, followed by a final analysis to determine the final material composition of each fraction. The analysis of fraction quality and the subsequent material recovery of the two characterization paths may help provide helpful insight. More importantly, the result will help identify the type of sample it corresponds to and compare 
characterization results over time and in different regions. In addition, the insight generated from the characterization analysis would help define further treatment options.

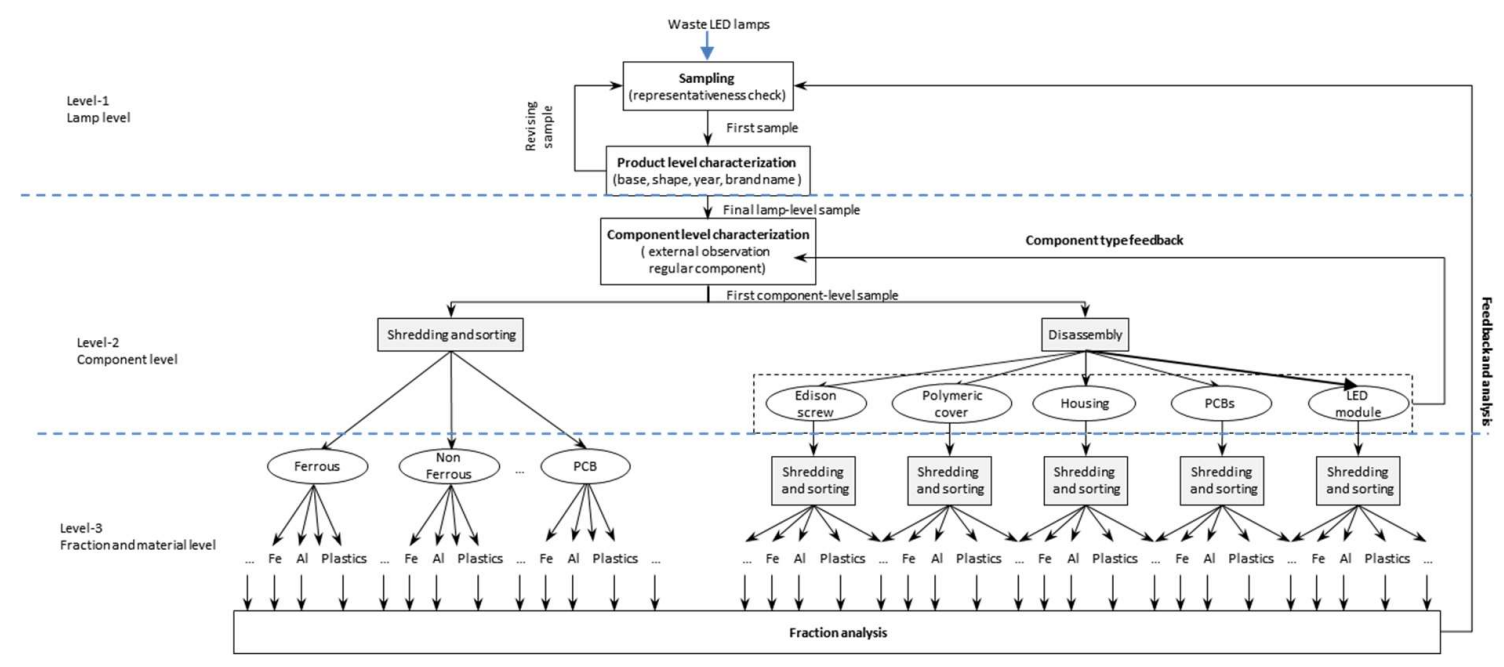

Figure 3. Characterization protocol proposed for consistency

\subsection{Conclusion:}

The paper aims at initiating a discussion for a more representative and consistent characterization process for LED lamps. The paper argues that current characterization rarely allows comparison among different studies, marked with wide variation and inconsistent reporting. In addition, the approach lacks intent in the documentation of the lamp-componentmaterial level composition, precluding extraction of helpful insight that is a prerequisite for developing a robust recycling line. A better understanding of the lamp-component-material composition nexus would help define a recycling line, more diverse and adaptive to the strategic and economic interests. To rip the benefits of the future characterization results, we have proposed a characterization protocol. The protocol is by no means a final work plan. It can be modified, upgraded, revised, and standardized to enhance smooth applicability.

The paper may stimulate future researchers to demonstrate additional insight by following this approach and upgrading it to the context. Finally, the future characterization studies are expected to be able to deliver a relatively consistent work plan to ensure the following: if changes of lamp level feature over time are needed to track; if the classification of lamp level has significant impacts on component and material level recovery; if changes in any direction would warrant adjustment of the recycling line; if understanding lamp level would enable recyclers early decision making

\section{Acknowledgments}

This research was funded by EIT RawMaterials as part of the project "REDLED: Recycling EnD-of-life LED" with the project number 18039. 


\section{References}

Cenci, M.P., Dal Berto, F.C., Camargo, P.S.S., Veit, H.M., 2021. Separation and concentration of valuable and critical materials from wasted LEDs by physical processes. Waste Management 120, 136-145. https://doi.org/10.1016/j.wasman.2020.11.023

Cenci, M.P., Dal Berto, F.C., Schneider, E.L., Veit, H.M., 2020. Assessment of LED lamps components and materials for a recycling perspective. Waste Management 107, 285-293. https://doi.org/10.1016/j.wasman.2020.04.028

Dzombak, R., Padon, J., Salsbury, J., \& Dillon, H. (2017). Assessment of end-of-life design in solid-state lighting. Environmental Research Letters, 12(8), 084013.

Hendrickson, C., Matthews, D., Ashe, M., Jaramillo, P., 2010. Reducing environmental burdens of solid-state lighting through end-of-life design. Environmental Research Letters 5, 014016. https://doi.org/10.1088/1748-9326/5/1/014016

Kumar A., Kuppusamy V. K., Holuszko M., 2018. Liberation in waste LED lamps using high pulse fragmentation, International Mineral Processing Council conference paper. September 15-21,2018, Moscow, Russia.

Kumar, A., Kuppusamy, V.K., Holuszko, M., Song, S., Loschiavo, A., 2019. LED lamps waste in Canada: Generation and characterization. Resources, Conservation and Recycling 146, 329-336. https://doi.org/10.1016/j.resconrec.2019.04.006

LED professional, 2016. LED Lamps Recycling Technology for a Circular Economy [WWW Document]. URL https://www.led-professional.com/resources-1/articles/led-lamps-recyclingtechnology-for-a-circular-economy (accessed 4.15.21).

Lim, S.-R., Kang, D., Ogunseitan, O., Schoenung, J., 2013. Potential Environmental Impacts from the Metals in Incandescent, Compact Fluorescent Lamp (CFL), and Light-Emitting Diode (LED) Bulbs. Environmental science \& technology 47.

https://doi.org/10.1021/es302886

Lim, S.-R., Kang, D., Ogunseitan, O.A., Schoenung, J.M., 2011. Potential Environmental Impacts of Light-Emitting Diodes (LEDs): Metallic Resources, Toxicity, and Hazardous Waste Classification. Environ. Sci. Technol. 45, 320-327. https://doi.org/10.1021/es101052

Martins, T.R., Tanabe, E.H., Bertuol, D.A., 2020. Innovative method for the recycling of endof-life LED bulbs by mechanical processing. Resources, Conservation and Recycling 161, 104875. https://doi.org/10.1016/j.resconrec.2020.104875

Rahman, M.S.M., Kim, J., Lerondel, G., Bouzidi, Y., Clerget, L., 2019. Value Retention Options in Circular Economy: Issues and Challenges of LED Lamp Preprocessing.

Sustainability 11, 4723. https://doi.org/10.3390/su11174723

Scdhec. 2021. Waste Characterization Guidelines. available at

https://scdhec.gov/sites/default/files/docs/HomeAndEnvironment/Docs/Waste\%20Characteriz ation--GuidanceDocument.pdf . accessed on 06/08/2021

Zamprogno Rebello, R., Weitzel Dias Carneiro Lima, M.T., Yamane, L.H., Ribeiro Siman, R., 2020. Characterization of end-of-life LED lamps for the recovery of precious metals and rare earth elements. Resources, Conservation and Recycling 153, 104557.

https://doi.org/10.1016/j.resconrec.2019.104557 
Zhan, L., Xia, F., Ye, Q., Xiang, X., Xie, B., 2015. Novel recycle technology for recovering rare metals $(\mathrm{Ga}, \mathrm{In})$ from waste light-emitting diodes. Journal of Hazardous Materials 299, 388-394. https://doi.org/10.1016/j.jhazmat.2015.06.029 